Research Paper

\title{
Propofol attenuates osteoclastogenesis by lowering RANKL/OPG ratio in mouse osteoblasts
}

\author{
Do-Won Lee ${ }^{1}$, Jae-Young Kwon ${ }^{\circledR}$, Hae-Kyu Kim ${ }^{1}$, Hyeon-Jeong Lee ${ }^{1}$, Eun-Soo Kim¹ ${ }^{1}$ Hyae-Jin Kim¹, \\ Hyung-Joon Kim², Han-Bit Lee ${ }^{1}$ \\ 1. Department of Anesthesia and Pain Medicine, Biomedical Research Institute, Pusan National University Hospital, Busan, Republic of Korea \\ 2. Department of Oral Physiology, BK21 PLUS Project, and Institute of Translational Dental Sciences, School of Dentistry, Pusan National University, Yangsan, \\ Republic of Korea \\ $\square$ Corresponding author: Jae-Young Kwon, Tel: +82-51-240-7399, Fax: +82-51-242-7466, Email: jykwon@pusan.ac.kr
}

(0) Ivyspring International Publisher. This is an open access article distributed under the terms of the Creative Commons Attribution (CC BY-NC) license (https://creativecommons.org/licenses/by-nc/4.0/). See http://ivyspring.com/terms for full terms and conditions.

Received: 2017.09.06; Accepted: 2018.04.12; Published: 2018.05.14

\begin{abstract}
Bone remodeling plays an important role in the bone healing process; for example, following fracture. The relative ratio of the receptor activator of nuclear factor kappa $B$ ligand (RANKL)/ osteoprotegerin (OPG) controls osteoclast differentiation, thereby playing a pivotal role in the regulation of bone remodeling. Propofol, a widely used anesthetic agent in orthopedic procedures, is considered to possess potential antioxidant properties owing to its structural similarity to a-tocopherol. Antioxidants are known to enhance bone healing. Accordingly, in the present study, we aimed to investigate osteoblastic differentiation and RANKL/OPG expression following propofol administration, in order to assess the potentially beneficial effects of this drug on the bone remodeling process, using calvarial primary osteoblasts from newborn mice. Calvarial pre-osteoblast cells were cultured in media containing clinically relevant concentrations of propofol, and cytotoxicity, effects on cell proliferation, osteogenic activity, and osteoclastogenesis were examined. The present findings indicated that propofol did not exert cytotoxic effects or alter cell proliferation in primary calvarial osteoblasts. Further, propofol did not affect osteoblast differentiation. The RANKL/OPG ratio was found to be decreased following propofol administration, and osteoclastogenesis was significantly reduced, indicating that propofol attenuated the osteoclastogenesis-supporting activity of osteoblasts. The results demonstrate that propofol, at clinically relevant concentrations, exerts beneficial effects on bone remodeling by attenuating osteoclastogenesis via suppression of the RANKL/OPG expression axis.
\end{abstract}

Key words: Bone remodeling; Osteoblast; RANKL; OPG; Propofol

\section{Introduction}

Bone homeostasis is maintained by a balance between bone remodeling, osteoblastic bone formation, and osteoclastic bone resorption [1-5]. In the context of the bone healing process, bone remodeling has been intensively studied and various cytokines, proteases, and morphogens have been reported to play important roles [6,7]. Among these bone biochemical markers, the receptor activator of nuclear factor kappa B ligand (RANKL), receptor activator of nuclear factor kappa B (RANK), and osteoprotegerin (OPG) molecular axis reflects the metabolic states of osteoblasts and osteoclasts $[8,9]$.
The prerequisite osteoclastogenic cytokine, RANKL, is synthesized and secreted by osteoblasts. More recently, a large number of studies have underlined the osteocyte as a major source of RANKL for regulating bone remodeling of postnatal bones [10-12]. The binding of RANKL to its membrane receptor RANK is pivotal to both initiation of osteoclast differentiation and osteoclast-mediated bone resorption [13,14]. OPG, a glycoprotein mainly synthesized by osteoblasts, acts as a circulating decoy receptor of RANKL [15]. In general, OPG binds to RANKL and blocks its activity, which results in the 
inhibition of osteoclast differentiation and subsequent bone resorption [16-18]. The relative ratio of RANKL/OPG controls the differentiation and function of osteoclasts and determines the bone remodeling status $[19,20]$.

Propofol is an intravenous anesthetic agent used for general anesthesia as well as for sedation in intensive care units. It is widely used for orthopedic surgery owing to the advantages of rapid onset, a short duration of action, and rapid elimination [21,22]. In addition to its sedative-hypnotic activity, propofol has anti-inflammatory and antioxidative effects $[23,24]$. Numerous previous studies have shown the beneficial effects of antioxidants in bone remodeling $[25,26]$. Therefore, it was presumed that the propofol treatment may enhance the bone remodeling process. Although several studies of the effects of various drugs on bone loss and fracture outcome have been reported $[27,28]$, the effect of propofol on bone remodeling process has not been clearly investigated.

In the present study, we aimed to examine the effect of propofol on osteoblastic differentiation and the expression of RANKL/OPG, using the calvarial primary osteoblast culture system, to elucidate the potential effect of propofol administration on the bone remodeling process.

\section{Materials and Methods}

\section{Reagents}

Propofol was obtained from Fresenius Kabi Austria $\mathrm{GmbH}$ (Hafnerstrabe, Austria). Leukocyte Alkaline Phosphatase Kit (for ALP staining), Leukocyte Acid Phosphatase Kit (for TRAP staining), $\beta$-glycerophosphate, and L-ascorbic acid were purchased from Sigma-Aldrich (St. Louis, MO). ALP enzyme activity was measured using the ALP Assay Kit from TAKARA BIO, Inc (Shiga, Japan). Soluble RANKL and M-CSF were purchased from PeproTech (Rocky Hill, NJ). All other chemicals and reagents were purchased from Sigma-Aldrich.

\section{Cytotoxicity and cell proliferation measurement}

The effects of propofol on cell viability and proliferation were measured by a colorimetric method utilizing the 3-(4,5-dimethylthiazol)-2,5-diphenyltetrazolium bromide (MTT, Sigma-Aldrich) assay. In brief, calvarial pre-osteoblast cells were plated in 96-well plates and treated with the indicated doses of propofol $(0,5,10,20,50,100 \mu \mathrm{M})$ for up to 3 days. At the end of the culture period, cells were incubated with fresh medium containing $0.5 \mathrm{mg} / \mathrm{ml} \mathrm{MTT}$ solution for over 4 hours. After incubation, blue formazan product formation was measured using a microplate reader at a wavelength of $570 \mathrm{~nm}$.

\section{Calvarial osteoblast preparation and osteogenic differentiation}

Primary calvarial osteoblasts from 1-day-old ICR mice were purified. The dissected calvaria were enzyme-digested in serum-free a-MEM (WELGENE, Daegu, Korea) containing $0.1 \%$ collagenase and $0.2 \%$ dispase for $15 \mathrm{~min}$ at $37^{\circ} \mathrm{C}$ with vigorous shaking. The digestion procedure was repeated five times. Purified calvarial pre-osteoblasts were seeded onto a 48-well plate and osteogenic differentiation was induced by culturing in a-MEM media supplemented with 10 $\mathrm{mM} \beta$-glycerophosphate and $100 \mu \mathrm{M}$ L-ascorbic acid (osteogenic media, OM). Osteogenic differentiation was evaluated by ALP staining or ALP enzyme activity assay according to the manufacturer's instructions.

\section{Bone marrow-derived macrophage (BMM) preparation and osteoclastogenic differentiation}

Five-week-old female ICR mice were sacrificed for femur and tibial extraction. Whole bone marrow cells were flushed out from tibiae and femurs of mice with serum-free a-MEM, and flushed cells were cultured in a 100-mm culture dish for 24 hours. After discarding adherent cells, the non-adherent cells were collected and further cultured in the presence of M-CSF $(30 \mathrm{ng} / \mathrm{ml})$ for 3 days. The bone marrow-derived macrophages (BMMs) obtained were used as osteoclast precursor cells for in vitro osteoclast differentiation studies. Osteoclast differentiation was induced by culturing BMMs in osteoclastogenic medium (a-MEM containing $30 \mathrm{ng} / \mathrm{ml} \mathrm{M-CSF}$ and $100 \mathrm{ng} / \mathrm{ml}$ RANKL) for 4 days. At the end of the culture period, mature osteoclasts were visualized by TRAP staining and TRAP-positive multinucleated $(\geq 4$ nuclei) cells were counted as osteoclasts.

\section{Conditioned media (C.M.) collection from osteoblast culture}

Calvarial osteoblasts $\left(2 \times 10^{6}\right)$ were seeded on 100-mm culture dishes and cultured for 7 days in osteogenic media $(\mathrm{OM}, 10 \mathrm{mM} \beta$-glycerophosphate + $100 \mu \mathrm{M}$ L-ascorbic acid) with propofol $(0,20,50 \mu \mathrm{M})$. On day 7, culture media were replaced by fresh a-MEM (without propofol). On the next day, conditioned media, (C.M.) were obtained. Collected C.M. were briefly centrifuged to eliminate cell debris and filtered using a $0.2-\mu \mathrm{m}$ pore-sized syringe filter before use.

\section{Osteoclastogenic activity assay using conditioned media}

In order to evaluate the osteoclastogenic activity 
of C.M. from osteoblasts, pre-osteoclast (pre-OC) differentiation was induced by culturing BMMs with M-CSF $(30 \mathrm{ng} / \mathrm{ml})$ and RANKL $(100 \mathrm{ng} / \mathrm{ml})$ for 2 days. The pre-OCs were treated with C.M. mixture (60 $\%$ C.M. + $40 \%$ fresh a-MEM without M-CSF \& RANKL) and further cultured for up to 3 days ( 5 days in total). After the 5-day (in total) culture period, cells were stained for detection of TRAP activity and TRAP-positive multinucleated ( $\geq 4$ nuclei) cells were counted as mature osteoclasts.

\section{Real-time PCR and RT-PCR}

Quantification of mRNA expression by PCR analysis was performed following a standard procedure. Total RNAs were purified with TRIzol reagent (Invitrogen), and $2 \mathrm{mg}$ of RNAs were reverse-transcribed with Superscript II (Invitrogen) according to the manufacturer' $s$ instructions. For real-time PCR analysis, cDNAs were amplified with SYBR green PCR master mix (Applied Biosystems) for 40 cycles of 15 seconds, denaturation at $95^{\circ} \mathrm{C}$, and 1 minute of amplification at $60^{\circ} \mathrm{C}$ using an AB7500 instrument (Applied Biosystems). The expression of RANKL and OPG mRNA levels were normalized against HPRT mRNA levels. The primer sequences used for PCR analysis were as follows: ALP, 5'-AC FTFFCTAAFAATFTCATC-3' (forward) and 5'-CTFFT AFFCFATFTCCTTA-3' (reverse); OCN, 5'-CATGAG AGCCCTCACA-3' (forward) and 5'-AGAGCGACAC CCTAGAC-3' (reverse); OPN, 5'-CCAAGTAAGTCC AACGAAAG-3' (forward) and 5'-GGTGATGTCCTC GTCTGT-3' (reverse); RANKL, 5'-CCAGCATCAAA ATCCCAAGT-3' (forward) and 5'-CCCCTTCAGATG ATCCTTC-3' (reverse); OPG, 5'-TGCAGTACGTCAA GCAGGAG-3' (forward) and 5'-TGACCTCTGTGAA AACAGC-3' (reverse); HPRT, 5'-CCTAAGATGATCG CAAGTTG-3' (forward) and 5'-CCACAGGGACTAG AACACCTGCTAA-3' (reverse).

\section{Statistical analysis}

Data are presented as the mean \pm SD of results from three independent experiments. The Student's $\mathrm{t}$-test was used to determine the significance of differences between two groups. Differences with $p<$ 0.05 were regarded as significant and denoted using an asterisk.

\section{Results}

\section{Propofol did not exert cytotoxic effects or alter cell proliferation in primary calvarial osteoblasts}

The effect of propofol on the cell viability of calvarial osteoblasts was examined using MTT assay. As shown in Fig. 1A, MTT assay results indicated that cell viability of calvarial osteoblasts was not affected by propofol administration. As shown in Fig. 1B, cell proliferation of calvarial osteoblasts increased with time, and the propofol concentration had no significant effect on this cell proliferation.

A

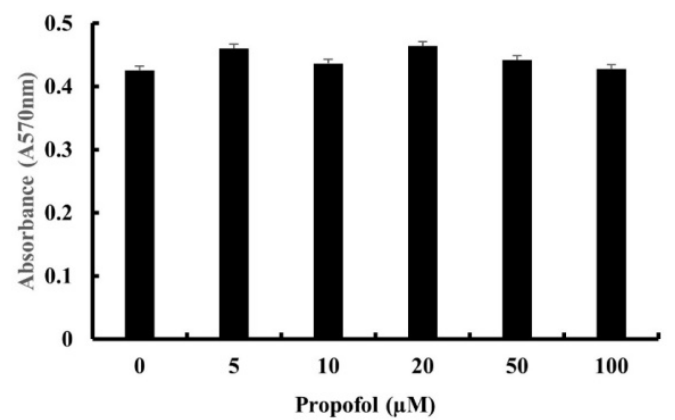

B

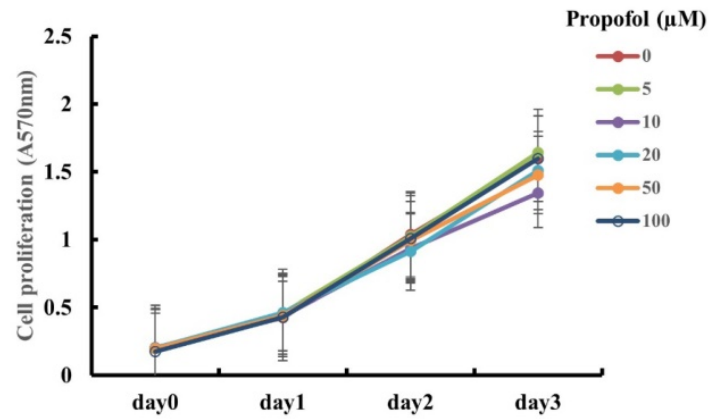

Figure 1. Propofol does not exert cytotoxic effects or alter cell proliferation in primary calvarial osteoblasts. (A) Calvarial osteoblasts were incubated in medium containing indicated concentrations of propofol $(0-100 \mu \mathrm{M})$ for 24 hours. Cell viability was evaluated by MTT assay. (B) Calvarial osteoblasts were cultured in osteogenic media (OM, $10 \mathrm{mM} \beta$-glycerophosphate $+100 \mu \mathrm{M}$ L-ascorbic acid) for 3 days in the presence of indicated doses of propofol (0-100 $\mu M)$. Cell proliferation was measured at daily intervals by MTT assay.

\section{Propofol did not affect osteoblast differentiation}

The effect of propofol on osteogenic differentiation of calvarial pre-osteoblast cells was evaluated by ALP staining and ALP enzyme activity assay. ALP staining, results indicated that osteoblast differentiation of calvarial pre-osteoblast cells was not affected by propofol administration (Fig. 2A). Calvarial pre-osteoblast cells were differentiated into osteoblasts in OM containing indicated concentrations of propofol $(0,5,20,50 \mu \mathrm{M})$. At days 0,4 , and 8 , the quantitative ALP enzyme activity assay was performed. As shown in Fig. 2B, there was no significant difference in osteoblast differentiation according to the concentration of propofol. 


\section{The RANKL/OPG ratio was decreased following propofol administration}

The effects of propofol on OPG and RANKL expression in calvarial pre-osteoblast cells were examined. Calvarial pre-osteoblast cells were cultured in OM for 8 days in the presence of indicated doses of propofol $(0,20,50 \mu \mathrm{M})$. At the end of the culture period, the mRNA levels of osteoblast markers (ALP, OCN, and OPN) and osteoclastogenic cytokines (RANKL and OPG) were examined by RT-PCR. While propofol induced little change of ALP, OCN, and OPN mRNA expression, a concentration-dependent decrease in RANKL mRNA expression and increase in OPG mRNA expression were evident (Fig. 3A). In addition, the expression levels of RANKL and OPG mRNA were assessed by quantitative real-time PCR analyses. As shown in Fig. 3B, propofol administration, at the concentration of $50 \mu \mathrm{M}$,

A

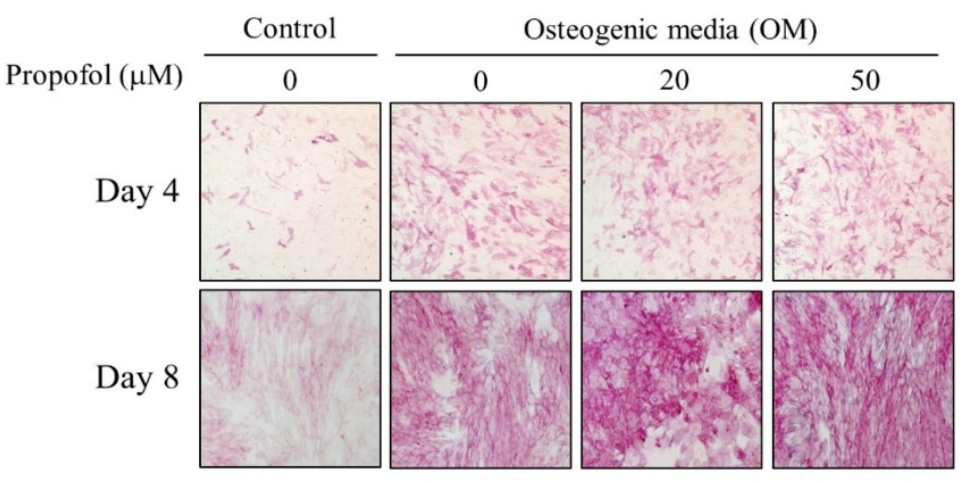

B

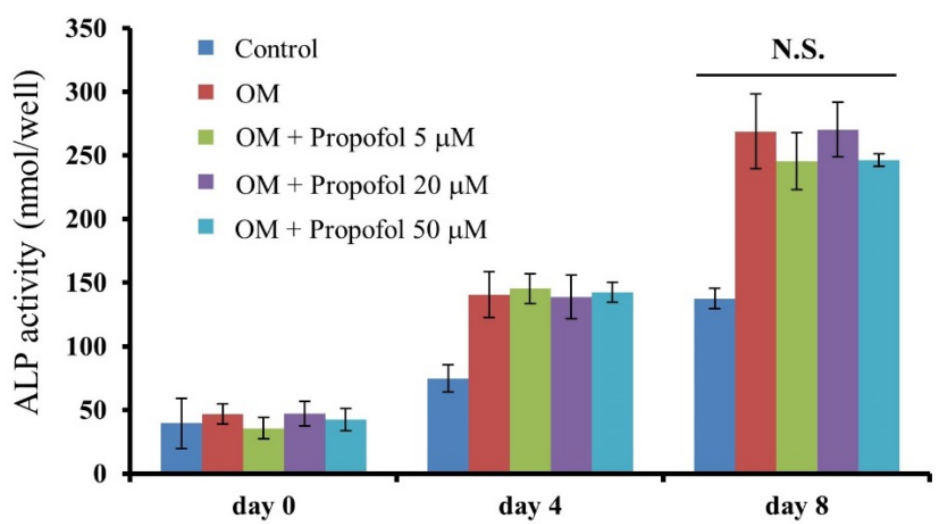

Figure 2. Propofol does not affect osteoblast differentiation. (A) Calvarial pre-osteoblast cells isolated from newborn mice were cultured in osteogenic media (OM, $10 \mathrm{mM} \beta$-glycerophosphate $+100 \mu \mathrm{M}$ L-ascorbic acid) for the indicated number of days. At day 4 and day 8 , osteoblast differentiation was examined by ALP staining. (B) Calvarial pre-osteoblast cells were differentiated into osteoblast in OM. At days 0,4 , and 8 , quantitative ALP enzyme activity assay was performed. significantly decreased RANKL expression 0.21-fold and increased OPF expression 3.90-fold compared with non-administration of propofol.

\section{Propofol lowered the osteoclastogenesis- supporting activity of osteoblasts}

The effect of propofol on the osteoclastogenesissupporting activity of osteoblasts was examined. A schematic diagram for C.M preparation is shown at the top of Fig. 4A. Then, osteoclast differentiation was induced from BMMs with M-CSF $(30 \mathrm{ng} / \mathrm{ml})$ and RANKL $(100 \mathrm{ng} / \mathrm{ml})$ for 2 days. At the pre-osteoclast stage (pre-OC), the culture media were replaced with C.M. (without M-CSF \& RANKL) and pre-OCs were allowed to differentiate into osteoclasts for 3 additional days. A schematic diagram showing osteoclast differentiation experiment is provided at the bottom of Fig. 4A. After 5 days of culture, TRAP staining was performed and the number of mature osteoclasts was counted to assess the effect of propofol on osteoclastogenesis. As shown in Fig. 4B, propofol administration at a concentration of $50 \mu \mathrm{M}$ significantly reduced osteoclastogenesis 0.39-fold compared with non-administration of propofol.

\section{Discussion}

Propofol is widely used for induction and maintenance of general anesthesia in various clinical situations [21,22]. In the present study, we aimed to investigate osteoblastic differentiation and RANKL/ OPG expression in the presence of propofol in order to assess the potentially beneficial effects of this drug on the bone remodeling process.

The first finding of this study was that propofol does not exert cytotoxic effects on, or affect the differentiation and proliferation of, osteoblasts. In the MTT assay, which was performed to evaluate the effect of propofol on osteoblast viability, the concentration of propofol used was as high as $100 \mu \mathrm{M}$. Further, the ALP enzyme activity assay for the evaluation of osteogenic differentiation, the concentration of propofol used was as high as $50 \mu \mathrm{M}$. Several previous studies reported that clinically relevant concentrations of propofol (approximately 17-62 $\mu \mathrm{M})$ had no harmful effect on osteoblasts and macrophages [29,30]. In accordance with previous reports, this study focused on propofol concentrations of $20 \mu \mathrm{M}$ and 
$50 \mu \mathrm{M}$, which lie within the range of clinically relevant concentrations. The present results were consistent with those of previous studies.
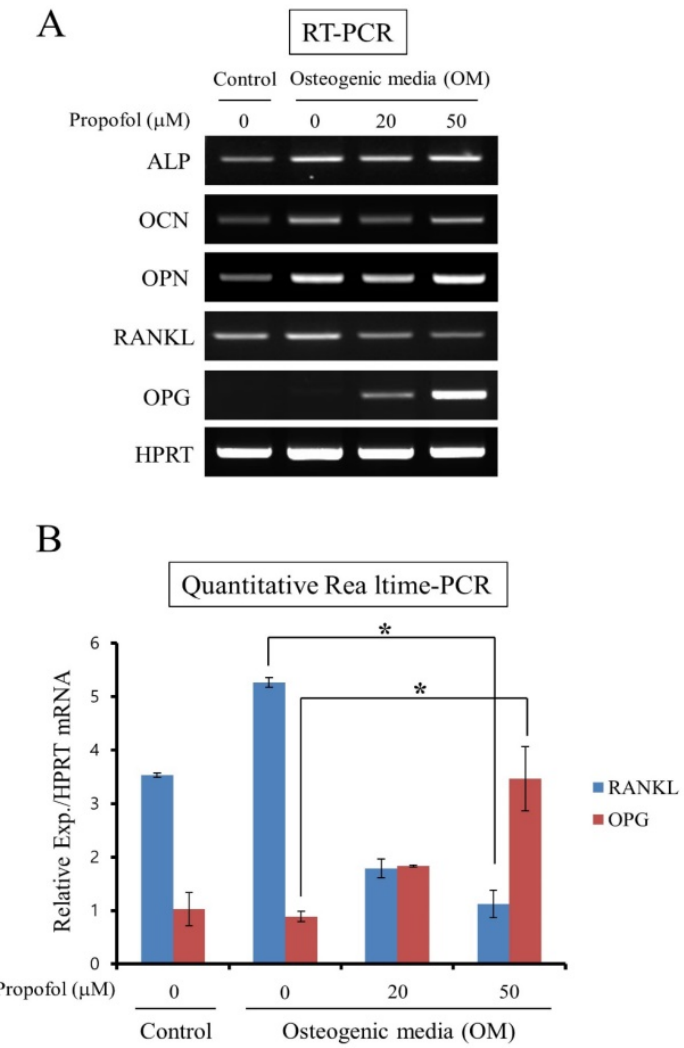

Figure 3. The RANKL/OPG ratio was decreased following propofol administration. (A) The mRNA expression levels of osteoblast markers (ALP, $\mathrm{OCN}$, and OPN) and osteoclastogenic cytokines (RANKL and OPG) were examined by RT-PCR. (B) The expression levels of RANKL and OPG mRNA were assessed by quantitative real-time PCR analyses. Quantitative data are means $\pm S D$ of results for three independent experiments $(*, p<0.05)$.

The second finding of this study was that the RANKL/OPG ratio was significantly decreased in the $50-\mu \mathrm{M}$ propofol-treated group, and OPG expression was increased in a propofol concentration-dependent manner. The synthesis and secretion of RANKL and OPG by osteoblasts plays a pivotal role in is bone remodeling [31]. The binding of RANKL to its receptor RANK, a member of the tumor necrosis factor receptor superfamily, triggers osteoclast differentiation [14,32]. Further, OPG, a member of the tumor necrosis factor receptor superfamily, functions as a decoy receptor for RANKL, thus inhibiting the interaction between RANKL and RANK by binding RANKL [13,14]. Interestingly, it has been shown that high serum RANKL levels are associated with fracture susceptibility, and high OPG levels are correlated with low risk of fracture [19,33]. Previous studies have reported that OPG deficiency causes bone loss, and OPG administration is effective in preventing bone resorption [34,35]. Recently, in addition to the individual effects of RANKL and OPG, the ratio of RANKL/OPG was suggested as an important determinant of bone remodeling during fracture healing [36]. As the RANKL/OPG ratio is relevant to osteoclast activity, increased RANKL/OPG ratio immediately after fracture is considered to affect bone resorption status in bone remodeling. Accordingly, strategies aimed at decreasing the RANKL/OPG ratio may be effective in enhancing the healing process of post-fracture $[14,37,38]$. It was hypothesized that propofol may reduce the RANKL/OPG ratio and affect osteoclast activity during bone remodeling. The results of the present study confirmed the efficacy of propofol, at a clinically relevant concentration $(50 \mu \mathrm{M})$, in reducing the RANKL/OPG ratio by decreasing RANKL expression and increasing OPG expression.

Further, the present study demonstrates that propofol administration at a clinically relevant concentration $(50 \mu \mathrm{M})$ significantly reduced osteoclastogenesis. Osteoclastogenesis comprised the following consecutive steps: differentiation of hematopoietic monocyte/macrophage lineage precursors to preosteoclasts, formation of osteoclasts, and bone resorption [39]. Critical molecules involved in osteoclastogenesis are RANK, RANKL, and macrophage-colony stimulating factor (M-CSF); the latter is additionally secreted by osteoblasts and stimulates RANK expression by binding to the M-CSF receptor $[13,14]$. The results of the present study showed that C.M from propofol-treated osteoblasts inhibited the fusion of mononucleated preosteoclasts to multinucleated osteoclasts. These findings suggest that propofol exerts anabolic effects on bone by inhibiting osteoclastogenesis.

However, several points must be addressed to maximize the beneficial effect of propofol on the bone remodeling process in clinical practice: importantly, propofol has no analgesic effect. Therefore, additional analgesia is essential when using propofol, as almost all orthopedic procedures are painful. Although the advantages of propofol in bone remodeling have been demonstrated, the maximum beneficial effect cannot be achieved when inappropriate analgesics are used together: this would result in the opposite effect, similar to that observed with non-steroidal antiinflammatory drugs [27]. Further studies must be performed to examine the effects of various kinds of analgesics on the bone remodeling process.

In conclusion, this study shows that propofol attenuates RANKL expression in osteoblasts without altering cell viability, indicating that propofol administration affects the bone remodeling process. As one of the most commonly used drugs in orthopedic procedures, propofol may be of utility owing to its observed beneficial effects on bone remodeling. 
A Conditioned medium (C.M.) preparation from osteoblasts

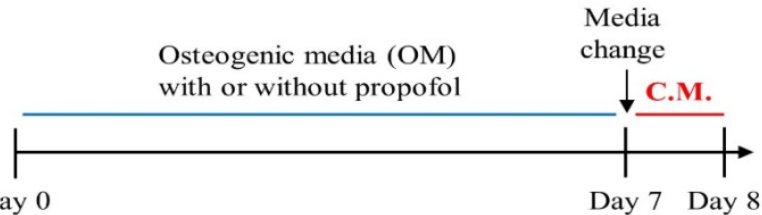

Osteoclast differentiation analyses

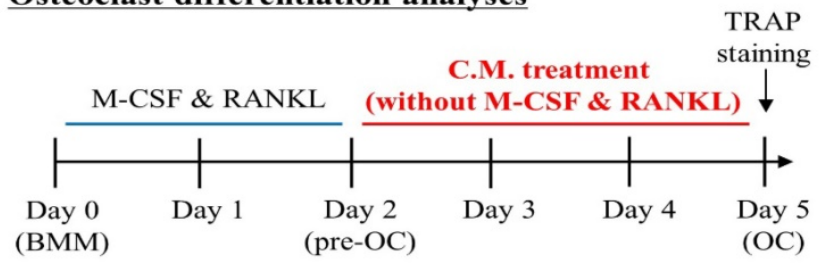

B C.M. Control OM only

$\mathrm{OM}+$

$\mathrm{OM}+$

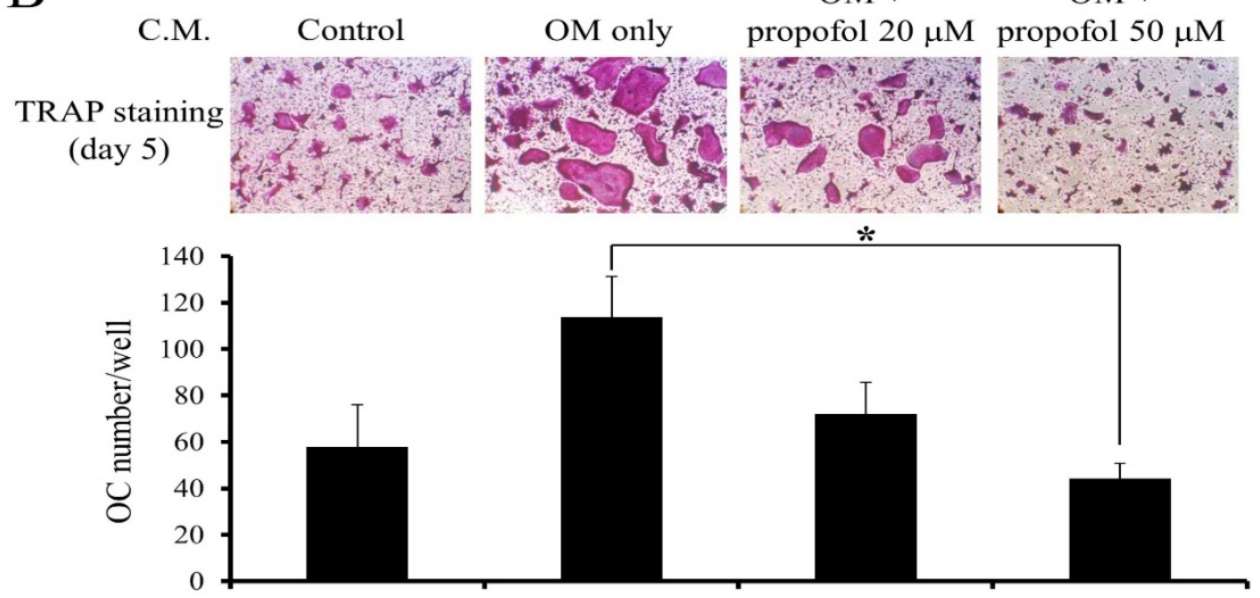

Figure 4. Propofol attenuates the osteoclastogenesis-supporting activity of osteoblasts. (A) The schematic diagram depicting the experiment (B) The osteoclastogenic activity of conditioned medium (C.M.) from propofol-treated osteoblasts was evaluated using an in-vitro osteoclast differentiation system. At the end of the culture period, cells were stained for TRAP activity and the numbers of osteoclasts/well were counted $(*, p<0.05)$.

\section{Supplementary Material}

Supplementary figures. http://www.medsci.org/v15p0723s1.pptx

\section{Acknowledgements}

This work was supported by clinical research grant from Pusan National University Hospital 2017

\section{Competing Interests}

The authors have declared that no competing interest exists.

\section{References}

1. Teitelbaum SL. Bone resorption by osteoclasts. Science 2000; 289: 1504-8.

2. Boyle WJ, Simonet WS, Lacey DL. Osteoclast differentiation and activation. Nature 2003; 423: 337-42.

3. Yamaguchi A, Komori T, Suda T. Regulation of osteoblast differentiation mediated by bone morphogenetic proteins, hedgehogs, and Cbfa1. Endocr Rev 2000; 21: 393-411.
4. Wozney JM, Rosen V, Celeste AJ, et al. Novel regulators of bone formation: Molecular clones and activities. Science 1988; 242: 1528-34.

5. Karsenty G, Kronenberg HM, Settembre C. Genetic control of bone formation. Annu Rev Cell Dev Biol 2009; 25: 629-48.

6. Einhorn TA. The cell and molecular biology of fracture healing. Clin Orthop Relat Res 1998; (355 Suppl): S7-21.

7. Gerstenfeld LC, Cullinane DM, Barnes GL, et al. Fracture healing as a post-natal developmental process: Molecular, spatial, and temporal aspects of its regulation. J Cell Biochem 2003; 88: 873-84.

8. Looker AC, Bauer DC, Chesnut $\mathrm{CH}$, et al. Clinical use of biochemical markers of bone remodeling: Current status and future directions. Osteoporos Int 2000; 11: 467-80.

9. Kurban S, Akpinar Z, Mehmetoglu I. Receptor activator of nuclear factor kappaB ligand (RANKL) and osteoprotegerin levels in multiple sclerosis. Mult Scler 2008; 14: 431-2.

10. Xiong J, Onal M, Jilka RL, et al. Matrix-embedded cells control osteoclast formation. Nat Med 2011; 17: 1235-41.

11. Xiong J, Piemontese $\mathrm{M}$, Onal $\mathrm{M}$, et al. Osteocytes, not osteoblasts or lining cells, are the main source of the RANKL required for osteoclast formation in remodeling bone. PLoS One 2015; 10: e0138189.

12. Nakashima $T$, Hayashi M, Fukunaga $T$, et al. Evidence for osteocyte regulation of bone homeostasis through RANKL expression. Nat Med 2011; 17: 1231-4.

13. Theill LE, Boyle WJ, Penninger JM. RANK-L and RANK: $T$ cells, bone loss, and mammalian evolution. Annu Rev Immunol 2002; 20: 795-823.

14. Lacey DL, Timms E, Tan HL, et al. Osteoprotegerin ligand is a cytokine that regulates osteoclast differentiation and activation. Cell 1998; 93: 165-76.

15. Simonet WS, Lacey DL, Dunstan CR, et al. Osteoprotegerin: A novel secreted protein involved in the regulation of bone density. Cell 1997; 89: 309-19. 
16. Schneeweis LA, Willard D, Milla ME. Functional dissection of osteoprotegerin and its interaction with receptor activator of NF-kappaB ligand. J Biol Chem 2005; 280 : 41155-64.

17. Hofbauer LC, Schoppet M. Clinical implications of the osteoprotegerin/RANKL/RANK system for bone and vascular diseases. JAMA 2004; 292: 490-5.

18. Masi L, Simonini G, Piscitelli E, et al. Osteoprotegerin (OPG)/RANK-L system in juvenile idiopathic arthritis: Is there a potential modulating role for OPG/RANK-L in bone injury? J Rheumatol 2004; 31: 986-91.

19. Abdallah BM, Stilgren LS, Nissen N, et al. Increased RANKL/OPG mRNA ratio in iliac bone biopsies from women with hip fractures. Calcif Tissue Int 2005; 76: 90-7.

20. Gravallese EM, Manning C, Tsay A, et al. Synovial tissue in rheumatoid arthritis is a source of osteoclast differentiation factor. Arthritis Rheum 2000; 43: 250-8.

21. Sebel PS, Lowdon JD. Propofol: A new intravenous anesthetic. Anesthesiology 1989; 71: 260-77.

22. McKeage K, Perry CM. Propofol: A review of its use in intensive care sedation of adults. CNS Drugs 2003; 17: 235-72.

23. Chen RM, Chen TG, Chen TL, et al. Anti-inflammatory and antioxidative effects of propofol on lipopolysaccharide-activated macrophages. Ann N Y Acad Sci 2005; 1042: 262-71.

24. Hsing $\mathrm{CH}$, Chou W, Wang JJ, et al. Propofol increases bone morphogenetic protein-7 and decreases oxidative stress in sepsis-induced acute kidney injury. Nephrol Dial Transplant 2011; 26: 1162-72.

25. Schelonka EP, Usher A. Ipriflavone and osteoporosis. JAMA 2001; 286: 1836-7.

26. Xu ZS, Wang XY, Xiao DM, et al. Hydrogen sulfide protects MC3T3-E1 osteoblastic cells against $\mathrm{H} 2 \mathrm{O} 2$-induced oxidative damage-implications for the treatment of osteoporosis. Free Radic Biol Med 2011; 50: 1314-23.

27. Gerstenfeld LC, Thiede M, Seibert K, et al. Differential inhibition of fracture healing by non-selective and cyclooxygenase- 2 selective non-steroidal anti-inflammatory drugs. J Orthop Res 2003; 21: 670-5.

28. Gerner P, O'Connor JP. Impact of analgesia on bone fracture healing. Anesthesiology 2008; 108: 349-50.

29. Chen RM, Wu GJ, Chang HC, et al. 2,6-diisopropylphenol protects osteoblasts from oxidative stress-induced apoptosis through suppression of caspase-3 activation. Ann N Y Acad Sci 2005; 1042: 448-59.

30. Chen $\mathrm{RM}, \mathrm{Wu} \mathrm{CH}$, Chang $\mathrm{HC}$, et al. Propofol suppresses macrophage functions and modulates mitochondrial membrane potential and cellular adenosine triphosphate synthesis. Anesthesiology 2003; 98: 1178-85.

31. Collin-Osdoby P, Nickols GA, Osdoby P. Bone cell function, regulation, and communication: A role for nitric oxide. J Cell Biochem 1995; 57: 399-408.

32. Yasuda H, Shima N, Nakagawa N, et al. Osteoclast differentiation factor is a ligand for osteoprotegerin/osteoclastogenesis-inhibitory factor and is identical to TRANCE/RANKL. Proc Natl Acad Sci U S A 1998; 95: 3597-602.

33. Schett G, Kiechl S, Redlich $\mathrm{K}$, et al. Soluble RANKL and risk of nontraumatic fracture. JAMA 2004; 291: 1108-13.

34. Mizuno A, Amizuka N, Irie K, et al. Severe osteoporosis in mice lacking osteoclastogenesis inhibitory factor/osteoprotegerin. Biochem Biophys Res Commun 1998; 247: 610-5.

35. Bekker PJ, Holloway D, Nakanishi A, et al. The effect of a single dose of osteoprotegerin in postmenopausal women. J Bone Miner Res 2001; 16: 348-60.

36. Wang XF, Zhang YK, Yu ZS, et al. The role of the serum RANKL/OPG ratio in the healing of intertrochanteric fractures in elderly patients. Mol Med Rep 2013; 7: 1169-72

37. Wasilewska A, Rybi-Szuminska A, Zoch-Zwierz W. Serum RANKL, osteoprotegerin (OPG), and RANKL/OPG ratio in nephrotic children. Pediatr Nephrol 2010; 25: 2067-75.

38. Coetzee M, Kruger MC. Osteoprotegerin-receptor activator of nuclear factor-kappaB ligand ratio: A new approach to osteoporosis treatment? South Med J 2004; 97: 506-11.

39. Corral DA, Amling M, Priemel M, et al. Dissociation between bone resorption and bone formation in osteopenic transgenic mice. Proc Natl Acad Sci U S A 1998; 95: 13835-40. 